\title{
Mélodies pleurées, paroles à attraper
}

Les chants d'əngurguro dans des funérailles éthiopiennes (Goğğam, Amhara)

\section{Katell Morand}

\section{OpenEdition}

1 Journals

Édition électronique

URL : https://journals.openedition.org/clo/592

DOI : $10.4000 /$ clo.592

ISSN : 2266-1816

Éditeur

INALCO

Édition imprimée

Date de publication : 30 juin 2011

Pagination : $57-78$

ISBN : 978-2-85831-197-2

ISSN : 0396-891X

\section{Référence électronique}

Katell Morand, « Mélodies pleurées, paroles à attraper », Cahiers de littérature orale [En ligne], 69 | 2011, mis en ligne le 17 janvier 2013, consulté le 03 juillet 2021. URL : http://journals.openedition.org/clo/ 592 ; DOl : https://doi.org/10.4000/clo.592

Ce document a été généré automatiquement le 3 juillet 2021.

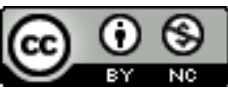

Cahiers de littérature orale est mis à disposition selon les termes de la Licence Creative Commons Attribution - Pas d'Utilisation Commerciale 4.0 International. 


\section{Mélodies pleurées, paroles à attraper}

Les chants d'əngurguro dans des funérailles éthiopiennes (Goğğam, Amhara)

\section{Katell Morand}

\section{L'annonce}

Dära mən täwäyayu Goğğam mən täwäyayu De quoi s'afflige-t-on au Dära, de quoi s'afflige-ton au Goğğam Wändəm wändəmun sigäläw əyayyu

On a vu un frère tuer son frère (Səmačäw, enterrement de Yəlaq, 6 décembre 2005)

1 La nuit tombe sur la vallée. Alors que des ombres blanches se glissent de maison en maison, la stupeur gagne partout les visages. Assise au ras du sol, à la lueur tremblante de sa lampe à huile, Fänta sursaute et se fige : les yeux grand ouverts, les bras relevés, et les mains croisées sur la tête, elle souligne de ses exclamations les paroles de son fils.

Yəlaq Aläme vient d'être poignardé par son frère cadet. Ce jeune voisin de vingt ans en est mort sur le coup tandis que son meurtrier s'enfuyait - et d'ailleurs, qui sait où il se cache et quelles sont ses intentions? Les abords du hameau bruissent de la confusion de cris et de pleurs des proches accourus d'un pas pressé; mais dans les maisons alentours, et alors que l'obscurité se fait plus dense, les discussions à mi-voix cèdent peu à peu la place au silence et à l'attente.

3 Bien que l'issue en soit rarement aussi violente, les conflits entre frères sont plutôt fréquents dans le Goğğam, une région des Haut-Plateaux du nord de l'Éthiopie ${ }^{1}$. Dans cette société rurale et agricole, ces conflits ont pour origine le partage problématique des droits sur la terre après le décès des parents : malgré des valeurs très égalitaires (des droits identiques pour tous les enfants), il existe - de fait - une compétition qui, 
accentuée par les récentes réformes agraires, tourne au désavantage des plus jeunes et nourrit leur colère ${ }^{2}$. Lorsqu'ils surviennent, les meurtres comme celui de Yəlaq en décembre 2005 entraînent une période de grande incertitude. En effet, parents et amis font alors face aux injonctions contradictoires des valeurs familiales : si d'un côté ils doivent à tout parent, y compris meurtrier, un soutien inconditionnel, leurs obligations les portent également à la vengeance. La réconciliation, c'est-à-dire la possibilité de reprendre des relations normales, est alors un processus long et douloureux.

4 J'ai choisi de présenter dans cet article des poèmes chantés lors des funérailles du jeune homme. Mon objectif est de montrer que les choix et les stratégies verbales des chanteurs sont décisifs pour la tournure que prendront les événements. Nous verrons en effet que l'efficacité pragmatique des chants d'əngurguro permet de construire une interprétation de la mort du jeune homme, et que celle-ci peut être déterminante, à long terme, pour les décisions et les actions des protagonistes.

\section{Mélodies pleurées : les chants d'əngurguro et leurs effets}

\section{Mouvements concentriques et superpositions sonores}

En fin de matinée, un long cortège s'avance sur les chemins escarpés qui mènent à l'église. Les habitants de la paroisse et des parents tout juste arrivés de localités voisines se pressent dans un long murmure mélodique autour des sœurs, des tantes, et des oncles de Yəlaq. La bouche couverte d'un pan de tissu, les yeux au sol et le corps affecté d'un léger balancement, ils présentent l'un après l'autre leurs exclamations désolées et le bourdonnement de leurs condoléances ; effleurent un bras ou s'inclinent, d'un geste ample, vers une épaule ; puis reprennent leur marche au son des plaintes qui suivent le corps.

6 À trois reprises, le cortège s'arrête sous de grands arbres pour former des cercles concentriques. Les femmes endeuillées, vêtues de capes noires, se serrent au milieu : les poignets croisés sur le haut du crâne, elles se balancent ou sautillent, s'agitent de sanglots, et brandissent des objets personnels du défunt. En un cercle dense autour d'elles, les autres femmes circulent lentement, dans le sens inverse des aiguilles d'une montre. Enfin les hommes, le fusil à l'épaule se déplacent le long d'un troisième cercle, tandis que des proches du défunt déambulent dans l'espace laissé libre, répétant inlassablement quelques formules monocordes : « Hélas, mon fils... Yəlaq n'est plus... » (wäyñe ləğe... Yəlaq qärä...). Ne manquent aujourd'hui que les prêtres, alignés en temps ordinaire un peu à l'écart face au corps, sous leurs ombrelles bariolées - mais Yəlaq, en raison de son jeune âge, est enterré sans liturgie.

7 De la foule monte à intervalles réguliers un brouhaha de pleurs mélodiques. À la manière d'un refrain, celui-ci ponctue les courts poèmes que chantent à tour de rôle une douzaine d'hommes et de femmes ${ }^{3}$; et malgré le mouvement continu et la saturation sonore, l'attention se porte tout entière sur la poésie chantée.

\section{Ongurguro : tours de chant et réactions codifiées}

8 Ne sont chantés aujourd'hui que des poèmes dits d'əngurguro, l'une des principales catégories de poésie chantée en amharique, et que l'on retrouve classée dans la 
littérature sous les appellations de "lamentation» ou de «chant de deuil» (Getie Gelaye, 2005)4. Il est vrai les poèmes d'əngurguro sont systématiquement présents dans les contextes funéraires ; mais il n'est pas le seul genre musico-poétique que l'on y entende, car deux autres le côtoient ; et il ne s'y limite pas, puisqu'on le retrouve dans d'autres circonstances. Comme nous le verrons, cette précision a son importance.

Plus que par son contexte de performance, l'əngurguro se caractérise par sa ligne mélodique «pleurée » : des formules répétitives, dont l'effet de tremblement est obtenu grâce à une technique s'apparentant à du yodel (Morand, 2010b, 28-30). D'ailleurs, " chanter de l'əngurguro » (angwäräggwärä) peut se dire tout simplement "pleurer » ou «dire en pleurant». Ce qui est "dit» est une suite de paroles dont le cœur est un distique à rimes finales, ici signalé par un retrait. Forme par excellence de la poésie amharique, le distique est censé condenser l'opinion de son énonciateur ${ }^{5}$; il est interprété et mémorisé - "attrapé » - par des auditeurs d'autant plus attentifs à sa chute qu'il est précédé d'une longue introduction dont les phrases, composées d'interjections et de formules nominales, sont entrecoupées de silences (ici, des barres obliques suivies de retours à la ligne) ${ }^{6}$.. Les auditeurs comblent ces silences par des plaintes et des exclamations codifiées - «malheur à moi!» ou «ma mère, mon monde !»- et concluent chaque intervention de formules mélodiques dont l'ensemble, comme je l'ai souligné, n'est pas coordonné.

J'ai choisi de présenter ici les interventions de trois femmes (numérotées de 1 à 9), devant l'enceinte de l'église. Il s'agit des toutes dernières performances, quand les hommes reviennent du cimetière, où ils y sont entrés seuls, et reconstituent leur cercle autour des femmes. Abäğğu et Alämayähu, deux habitantes de la paroisse, sont les premières à alterner ${ }^{7}$ :

\section{Abäğğu}

Awäy awäy ənä Mälälo ənä əmäyte Särqe ənä วmäyte Täbayən/

Awäy awäy les gens de Mälälo, les gens de Dame Särqe, les gens de Dame Täbayən/

วnen yəbəlañ ənä Aläme Asfärd ənä əmäyte Alga/

Plutôt moi, que je sois mangée, les gens de Aläme Asfärd, les gens de Dame Alga/

วnä Wändəfraw Sənnəde Wändəfraw Mäkonən Wändəfraw/

Les gens de Wändəfraw, Sände Wändəfraw, Mäkonən Wändəfraw/

Ləğ Gäbre Gärrämaw/

L'enfant Gäbre Gärrämaw/

Täsäbasäbaw näw mutən mäqäsqäs/

Il faut se rassembler, secouer [le souvenir des] morts/

Tru ləğ antä/

Toi, bon enfant/

วnä วmäyte Šašä akalate wa/

Les gens de Dame Šašä/

Täsäbasäbaw näw mutən mäqäsqäs

Il faut se rassembler, secouer [le souvenir des] morts

ənämäyte atkəlt əyalu malqäs

« Les gens de Dame Atkəlt », disent-ils en pleurant

\section{2. Alämayähu}

Yänä วmäyte ləğ yäna Alga ləğ yä Gätəye ləğ/

Les gens du fils de ma Dame, les gens du fils d'Alga le fils de Gätəye/

Yänä วmäyte yänä วmäyte yänä วmäyte ləğ yäna Alga ləğ/

Les gens de ma Dame, les gens de ma Dame, les gens du fils de ma Dame, les gens du fils d'Alga/ 
Gäna yalbälaw gäna yalt'ät't'aw gäna lə̆̆/

Celui qui n'a pas encore mangé, pas encore bu, l'encore enfant/

Naq adrəgäw naquh balənğäročəhə

Ils te méprisaient, tes camarades

Käziya käč'äwataw kätəmhərt biyat'uhə

À présent ils t'ont perdu pour le jeu, pour l'école

\section{Abäğğ}

วnä mäna ənä mäna ənä mänaw bet/

Les habitants de cette vaine, de cette vaine maison/

วndäzəh bozänäh ləğe Abba Yəgəzaw akalate wa/

Ainsi tu as cessé le travail, mon enfant, Grand-père Yəgəzaw, mes chers corps, ah !/

Anbərbər əngäda ənä mäna/

L'invité saccageur, les habitants de la vaine [maison]/

Ato Yolqal yalbälaw gäna yalt'ät'aw gäna/

Monsieur Yolqal, celui qui n'a pas encore mangé, pas encore bu/

Ato Bäyə ləğ Gäbre Gärrämä/

Monsieur Bayə, l'enfant Gäbre Gärrämä/

Awäy awäy ənä mänaw bet/

Awäy awäy les gens de cette vaine maison/

amäyte Tagäz akalate wa/

Dame Tagäz, ah, mes chers corps !/

Awäy awäy วnämäyte Šašä/

Awäy awäy, les gens de Dame Šašä/

วnä วmäyte awäy/

Les gens de ma Dame, awäy/

Ahun käzih ləqso Šašätu bänoräč

Si à cet enterrement Šašätu était présente

วndä ənğäraw gagəra taqärbaw näbbäräč

Tout comme elle préparait la galette, elle l'aurait amené [sur un plateau] ${ }^{8}$

Les femmes en deuil, au centre, s'agitent de plus en plus; on entend des appels (« ̌̌ašä!»). Dans les autres cercles, les auditeurs, le regard perdu dans le vide, se couvrent la bouche et pleurent en se balançant légèrement.

\section{Abäğğu}

ənä Fətfət ənä əmäyte alga/

Les gens de Fətfət, les gens de Dame Alga/

วnä วmäyte Qolle/

Les gens de Dame Qollé/

ənä Wändəfraw ənä Wudde Dañaw/

Les gens de Wändəfraw, les gens de Wudde Dañaw/

Awäy yəbəlañ ənä əmäye wa /

Awäy, que je sois mangée, les gens de ma Dame, ah !/

ənä Wändəfraw ənä Aläme Asfärd/

Les gens de Wändəfraw, les gens de Aläme Asfärd/

Awäy awäy ləğ Yəlqal/

Awäy, awäy l'enfant Yəlqal/

orä dähna šäggaw tämari/

arä, le bon, le joli écolier/

ənä əmäyte Atəkəlt ənä əmäyte Särqew/

Les gens de Dame Atkəlt, les gens de Dame Särqew/

วnä əmäyte Täbayən/

Les gens de Dame Täbayən/

Akalate wa ənä Bälaynäš/

Ah, mes chers corps ! Les gens de Bälaynäš/ 
Yäzih bet säw/

Les gens de cette maison

Awäy yäzih bet säw biwəlm ayadär/

Awäy les habitants de cette maison, alors qu'ils ont passé la journée ne restent pas

la nuit/

วnä Gwad Aläm ənate lə̆̆g/

Les gens de Compagnon Aläm, le fils de ma mère/

Alqsobəñal alčalkuləšəm /

Il a pleuré sur moi, je ne pouvais rien pour toi (f)/

Awäy yäzih bet säw biwəlm ayadär

Awäy les habitants de cette maison, alors qu'ils ont passé la journée ne restent

pas la nuit

วndä bäga zänab yət'ät'al mədər

La terre les boit comme la pluie d'été

Mulu, qui habite une localité distante, décide alors de prendre son tour de chant:

5. Mulu

Awäy yännate lə̆̆, yä Alam lə̆̆/

Awäy, le fils de ma mère, le fils de Aläm/

Ləğ yä Aläm Asfärd Ato Yəlqal/

Le fils de Aläm Asfärd, Monsieur Yəlqal/

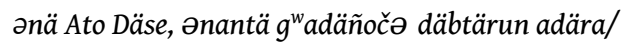

Les gens de Monsieur Däse, vous les amis il a laissé son cahier à vos soins/

วnantä g $g^{w}$ adäñoču däbtärun adära

Vous, ses amis, il a laissé son cahier à vos soins

Yəlqal hedw al agäñtot mäkära

Yəlqal est parti, il a rencontré le malheur

À ces mots, les femmes en deuil commencent à se frapper rythmiquement la poitrine ;

puis s'arrêtent quand Abäğğu reprend :

\section{Abäğğu}

วndämən näh yämutoč wägän/

Comment vas-tu, toi le partisan des morts/

ayä Ato Yəlaq zərzər Yəlaq antä mərt' zär awäy awäy/

Ayä Monsieur Yəlaq, épi à peine formé, toi Yəlaq excellente descendance, awäy

awäy/

Mən yazawärwal qalen əndä wänfit əndä wänfit

Qu'est-ce qui agite mes mots comme un tamis, comme un tamis

Amayäšən əmbi saləlat wädäfit

Avant que je lui dise «non, reviens », elle est partie

\section{Mulu}

Ato Yəlaq yäne zär bayläyəñ/

Monsieur Yəlaq, les gens de ma descendance, que je ne sois pas séparée/

Yəh Aläm zär bayläyəñ yəč Aläm Asfärd/

Cette descendance de Aläm, que je ne sois pas séparée, cet Aläm Asfärd/

Ato Yəlqal əhət wändəmoče ənä Ato Däse zär yämiyabäqəlut/

Monsieur Yəlqal, mes sœurs mes frères, les gens de Monsieur Däse qui plantent des

descendances/

Wägän/

Partisan/

Bäənnatu bäabbatu mutu yäbäzäbät

Chez sa mère, chez son père, les morts sont trop nombreux

Bäwaza wäddäqä gäna saydärsubät

Il est tombé soudainement, avant atteindre la maturité

\section{Alämayähu}


Yä วmäyte ləğ ayä ənnat aläm yä วmäyte ləğ əmäyte วmäyte/

Le fils de ma Dame, ayä, ma mère mon monde, le fils de ma Dame, ma Dame, ma

Dame/

Dähna g ${ }^{w}$ obäz mämot dähna qonğo mämot zarem täğämärä/

La mort des bons et braves, la mort des bons et beaux a commencé aujourd'hui/

Ayä ayä Ato Yəlaq ənnat aläm วmäyte/

Ayä, ayä Monsieur Yəlaq, ma mère mon monde, ma Dame

Dähna $g^{w}$ obäz mämot dähna qonğo mämot zarem täğämärä täğämärä

La mort des bons et braves, la mort des bons et beaux a commencé aujourd'hui, a commencé

Yänä T'ru lätə yänä Särqe lätə ənawqäw näbärä

L'époque de T'ru, l'époque de Särqe, nous la connaissions

Yäna Täbayənə ənawqäw näbärä ənawqäw näbärä

L'époque de Täbayən, nous la connaissions,connaissions, nous la connaissions

\section{Mulu}

Ayä əhət wändəmočə/

Ayä, mes sœurs, mes frères/

Yolqal Aläme/

Yəlqal Aläme/

วnä Gärämäw wändəmočə ənä tämari/

Les gens des frères de Gärrämäw les gens de l'écolier/

Agäre təz alläñ ašäbər/

J'ai le souvenir de mon pays, le terrifiant/

Tämari ləğ abäba/

Écolier, enfant, fleur/

Wändəmoče wa/

Mes frères, ah!/

Yač mät't'ač ləğ Aläm Asfärd yəbəlañ/

Celle-là est venue, fils de Aläm Asfärd, que je sois mangée/

วnä əmäyte sänəde yänä əğəgu Aläme/

Les gens de Dame Sänəde les gens de əğəgu Aläme/

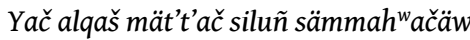

Cette pleureuse-là est venue, les ai-je entendus me dire

Biyalqubəñ näw ənği šäččče albällah ${ }^{w}$ ačäw

Mais c'est parce qu'ils me sont disparus, je ne gagne pas mon pain en les vendant

Les chanteuses sont alors interrompues; les cercles se défont en silence et tous s'alignent pour la prière qui clôturera le rituel.

\section{« Vous, les partisans » : toucher l'audience}

21 Que se passe-t-il donc au cours de la performance? Les pleurs s'amplifient et gagnent l'ensemble des participants, y compris les plus en retrait. Pour comprendre ce que font les chanteuses, il nous faut regarder en détail ce qui est dit.

\section{Nommer les morts : Yəlaq et sa parentèle}

Un premier aspect, peut-être le plus saisissant, est l'accumulation de noms propres : la quasi-totalité des introductions et une partie des distiques sont consacrées à des énumérations de défunts, tous désignés par un titre de respect («Dame», "Monsieur »). Cette accumulation a pour objectif d'augmenter la charge émotionnelle en ravivant d'anciennes douleurs, comme l'affirment explicitement les métadiscours d'Abäğğu (1) et d'un précédent chanteur : 
วnba ənkwan aywärdəm länandu säw biyalqəsu

La larme ne coule même pas quand on ne pleure qu'une personne

Yanəm yanəm əyäqäsäqäsu

De ceux-là et de ceux-là, ils secouent le souvenir distributif, c'est-à-dire qu'il désigne un ensemble d'individus partageant une même qualité, en l'occurrence un lien personnel avec le défunt. En invoquant un mort, les chanteuses interpellent donc ses partisans dans l'assemblée, sans que ceux-ci ne soient jamais nommément désignés. 
Pour cela les chanteuses doivent se plier à deux exigences :

- La première est que les défunts qu'elles mentionnent doivent faire partie de leur propre parentèle. Elles insistent ainsi, tout au long de leurs performances, sur leur position énonciative - c'est-à-dire d'où elles parlent. Grâce à l'usage répété des pronoms personnels et des termes de parenté ${ }^{10}$, elles affirment leurs liens avec ces défunts et, par conséquent, avec Yəlaq lui-même. Que Mulu (9) se défende d'être venue chanter en étrangère - même si l'accusation qu'elle rapporte n'est probablement qu'un effet de rhétorique - montre bien l'importance de cette implication personnelle.

- La seconde est de choisir des défunts qui toucheront le plus grand nombre d'auditeurs potentiels : mentionner un défunt dont elles sont proches, mais que personne ne connaît n'aurait aucune efficacité pragmatique. Leurs possibilités dépendent donc tout d'abord de leur proximité généalogique avec Yəlaq, et ensuite de leur connaissance des généalogies des autres membres de l'assemblée. Abäğğu, par exemple, peut tracer son lien avec Yəlaq par de multiples intermédiaires puisqu'elle en est une parente à la fois par son père Aläme et par sa mère Alga. Par ailleurs, comme elle habite dans la paroisse, elle est capable de croiser sa généalogie avec celles de ses voisins, d'où le nombre élevé de noms à sa disposition et la longueur de ses introductions. Par contraste, Alämeyəhu est une parente éloignée de Alga qui vit dans la paroisse sans en être originaire; elle ne dispose donc que d'un nombre très réduit de noms. Enfin, Mulu, apparentée à Aläme, est quasiment inconnue dans l'assemblée et doit faire appel à des ancêtres bien plus éloignés (comme Däse), mais dont des descendants sont susceptibles d'être présents. Chacune tente donc de toucher les membres de l'assistance en montrant la superposition de son propre réseau de partisans avec celui de Yəlaq d'une part, et ceux, implicites, des membres de l'assemblée d'autre part.

Pour autant, la nature exacte de ces liens n'est jamais explicitée : alors que la distance généalogique est essentielle dans les relations entre individus, elle est ici abolie par un usage générique des termes de parenté - «frère » (wändəm), « mère » (ənnat), etc. - et par la formulation ambiguë des connexions ${ }^{11}$. Cette manipulation se retrouve à l'occasion dans le langage ordinaire lorsqu'un locuteur désire accentuer la proximité avec l'un de ses parents; elle est ici systématique, comme si tous les partisans étaient réunis sur un plan sans nuances. Je ne prendrai ici qu'un seul exemple, celui de Särqe, dont le nom est mentionné à plusieurs reprises : bien que ses liens avec Yəlaq, Abbägu, Alämayähu, et divers membres de l'assemblée aient été de natures différentes, elle est ici, à un même titre, leur " partisane ». La seule distinction qui subsiste est celle du foyer (la «maison »); mais là encore, les formulations sont souvent ambiguës ${ }^{12}$.

Cet effacement des distances généalogiques par le discours est tout à fait comparable à celui qui s'opère dans l'espace concentrique des funérailles: les différences entre partisans (parents proches et lointains, amis, voisins, affins) sont abolies au profit des distinctions entre hommes et femmes ainsi qu'entre foyer (dans un sens très large) et autres partisans. Comme le montre la figure ci-dessous, la projection des défunts sur l'espace des vivants fait apparaitre d'autant plus clairement les intersections entre les réseaux personnels de Yəlaq, ceux des trois chanteuses, et potentiellement, ceux des membres de l'assemblée. 


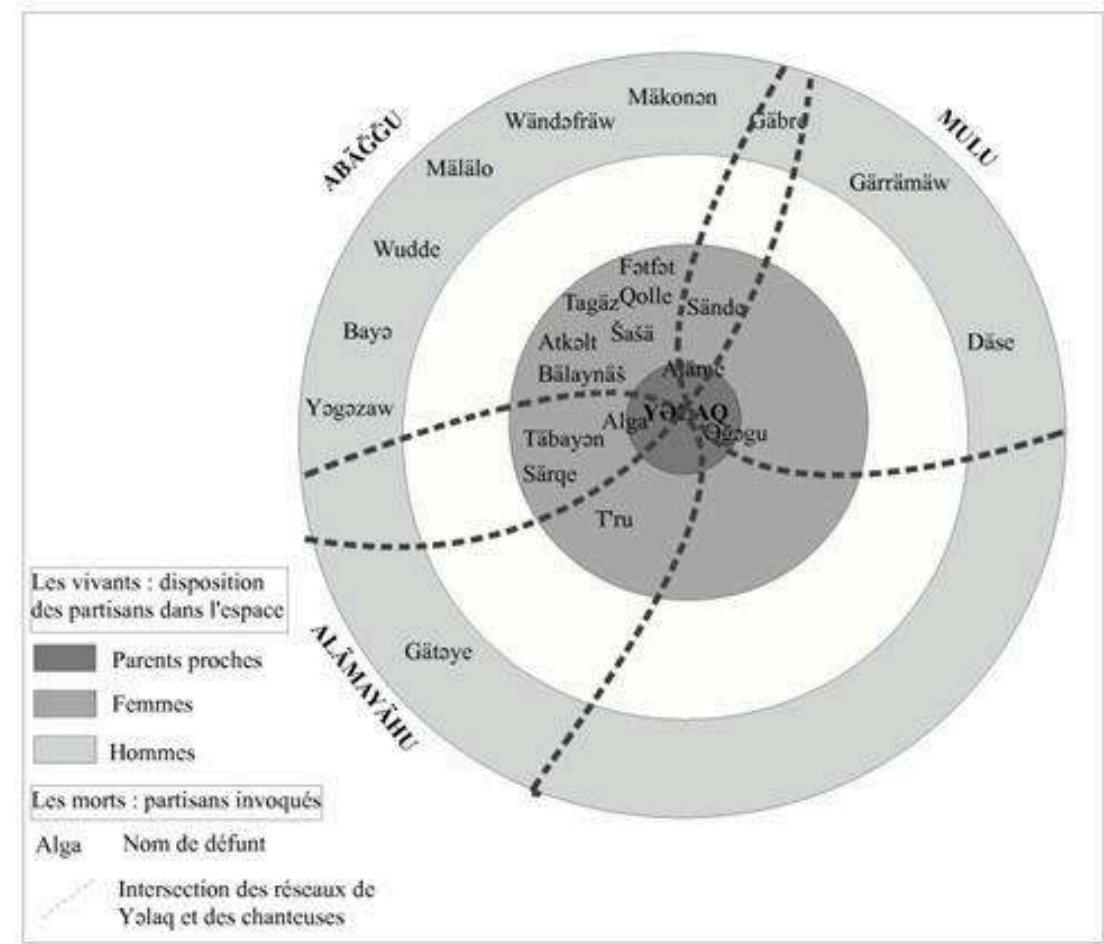

Intersection des réseaux de Yəlaq et des chanteuses dans l'espace des funérailles ${ }^{13}$ destinataires des énoncés. D'une ligne voire d'un groupe de mots à l'autre, les chanteuses s'adressent à Yəlaq, à d'autres défunts puis à des fractions de l'assemblée (" les gens de », "vous ses amis »), puis de nouveau à Yəlaq, etc. Ces changements ont pour effet un balancement entre la deuxième et troisième personne : on le voit, par exemple, dans la mise en scène des relations de Yəlaq et de ses amis : dans un premier distique (2), Alämayähu s'adresse ainsi au mort pour lui parler de ses camarades («Ils te méprisaient »), tandis que dans un second (5), Mulu s'adresse à ces mêmes camarades à propos de Yəlaq (« il a laissé son cahier à vos soins ») ${ }^{14}$.

Ensuite, des locuteurs tiers sont mobilisés dans des discours rapportés, sous la forme de la troisième personne du pluriel (« Ils disent que tu étais terrifié, étais-tu terrifié ?», chante un homme un peu plus tôt). Les noms de ces individus ne sont jamais précisés, 
ce pourrait s'apparenter au on-dit et à la rumeur ${ }^{15}$, mais il s'agit explicitement de participants aux funérailles. En leur prêtant des paroles, les chanteuses établissent dans le discours des relations entre elles-mêmes, certains membres de l'assistance, et des défunts, comme le montre cet exemple de Mulu (9) : «Cette pleureuse est venue, les ai-je entendus me dire/ Mais c'est parce qu'ils me sont disparus, je ne gagne pas mon pain en les vendant ».

En outre, des suffixes pronominaux apposés sur les verbes sont utilisés comme des marqueurs d'intentionnalité : -lä et -bä, suivis d'un pronom, indiquent pour le premier la faveur accordée, et pour le second le détriment ${ }^{16}$. Ces suffixes mettent eux aussi en évidence les liens entre les différentes personnes de l'énonciation («je », « tu », «il/ elle ») en insistant sur l'attention et le souci de l'autre, comme dans cet exemple d'Abäğğu (4) : « Il a pleuré sur moi, je ne pouvais rien pour toi (f)».

Ces trois premiers procédés accentuent l'effet d'entrecroisement des liens tout en rappelant les valeurs qui sont celles de la parenté : le souci mutuel, l'affection, et une entraide indéfectible. L'évocation de Šašä (3) est ainsi une occasion de célébrer la solidarité sans faille qui était la sienne - puisqu'elle aurait ramené Yəlaq de la mort ; les amis, en revanche, aurait négligé le jeune homme de son vivant, et sont rappelés aux obligations qui sont les leurs.

Enfin, le « je » de l'énonciatrice cède parfois la place à :

- un locuteur indéterminé, qui sous la forme d'un infinitif tient des paroles d'autorité sur les actions collectives à mener ${ }^{17}$;

- un "nous", qui est celui de l'assistance, ou plutôt de cette fraction de l'assistance interpellée par le nom d'un défunt ${ }^{18}$.

Ce dernier procédé est intéressant, car il crée autour de Yəlaq un sentiment de groupe et de solidarité là où il n'existe qu'une multitude de relations duales : les innombrables obligations réciproques liant les membres de l'assemblée les uns aux autres peuvent se fondre dans une même douleur.

Ces procédés mis en œuvre dans le discours ont donc une efficacité pragmatique : le réveil des anciens deuils, l'interpellation des individus, l'entrecroisement de leurs liens personnels, et la réaffirmation d'une certaine idéologie de la parenté touchent l'ensemble des auditeurs, créent un sentiment de solidarité, et les amènent à partager les pleurs de la famille proche. L'assistance est alors attentive à l'interprétation qui est donnée de la mort du jeune homme.

\section{« La terre les boit comme la pluie d'été » : une autre version de la mort de Yəlaq}

40 Le discours des chanteuses inclut tous les individus, avons-nous dit. Il est pourtant un protagoniste de cette histoire qui n'est jamais mentionné : le frère meurtrier. De fait, comme nous allons le voir, le meurtre est complètement occulté dans la version de la mort construite dans les poèmes.

\section{Une mort naturelle : « l'enfant Yəlaq » et son destin familial}

41 Commençons par le portrait qui est fait de Yelaq. Celui-ci est tout à fait surprenant : de bout en bout, il est dépeint comme un enfant. Il est vrai que l'absence de liturgie 
souligne qu'il n'était pas un adulte à part entière ${ }^{19}$; mais ce jeune homme avait tout de même dépassé depuis longtemps l'âge des jeux enfantins.

Comment les chanteuses y parviennent-elles? Elles procèdent, semble t-il, par des glissements de vocabulaire : en effet, si le mot ləğ (« enfant»), est omniprésent, c'est aussi parce qu'il s'agit d'un terme de parenté («fils» ou « fille»); et par ailleurs, dans les conversations ordinaires, il peut désigner tout individu dont on veut signaler la jeunesse, même si celle-ci n'est parfois que relative (à une situation donnée). De la même façon, l'épithète « écolier » (tämari) n'implique pas toujours l'enfance : dans ces zones rurales où l'éducation secondaire est très peu répandue, il peut être employé comme un titre de respect même pour une personne qui a abandonné ses études, comme c'était le cas de Yəlaq (qu'un autre chanteur appelle "le paysan au stylo »). C'est l'usage répété de ces termes, et leur combinaison avec l'évocation des jeux, l'image de la fleur, ou des adjectifs comme «joli», qui créent ce portrait d'un jeune enfant qui n'a pas eu le temps de grandir - qui n'a «encore » ni «mangé », ni «bu » (3). Les chanteuses affirment donc avec force que nous assistons non pas à l'enterrement d'un homme en âge de s'établir et de cultiver des relations complexes (y compris conflictuelles) avec son entourage, mais à celui d'un enfant - et ce, alors même que le nombre de personnes à s'être déplacées, l'élaboration des introductions chantées, et une certaine retenue dans la démonstration de la douleur (en particulier de la part des hommes), nous montrent tout le contraire.

Mais Yəlaq n'est pas qu'un jeune garçon mort trop tôt : son décès s'inscrit dans une série qui affecte l'ensemble de sa parentèle. Ses parents maternels et paternels, tous ceux dont les noms sont invoqués dans les introductions, sont ainsi présentés, quel que soit l'âge de leur mort, comme les victimes d'une fatalité s'abattant sur cette « maison vaine » (c'est-à-dire infertile) qui a vu disparaître en quelques années la plupart de ses habitants (3 et 7).

Plusieurs images nous dessinent les contours de cette fatalité. Celles de l'épi de blé, de la pluie trop fine pour rester à la surface de la terre ou des invités qui partent avant la nuit soulignent l'inaccomplissement; celle des fleurs sans fruits y ajoute un jeu d'opposition entre présent et passé révolu, comme dans cet autre distique d'Abäğğu :

Mätrom amälu näw yäzih bet azmära

Autrefois elles étaient connues, les moissons de cette maison

Abäbaw näw ənği frew aybäla

Il y a bien des fleurs, mais on ne mange pas les fruits

Cette image fait d'autant plus sens que le terme même de zär (la filiation ou descendance), qui signifie littéralement " semence », est déjà une métaphore végétale ${ }^{20}$. D'autres images établissent une comparaison avec les cycles naturels, tels que l'image de la course de la lune :

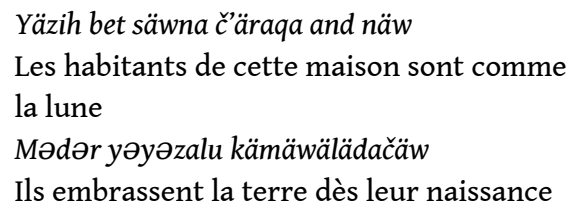

La fatalité qui pèse sur Yəlaq semble donc relever de l'ordre de la nature: toute causalité humaine en est ici écartée, comme si sa mort était entièrement indépendante du jeu des relations sociales. 


\section{Les vivants : impuissance et résignation}

47 Les causes de la mort de Yəlaq (et de ses parents) échappent donc au contrôle des humains, ce que les chanteuses soulignent en multipliant les indices de l'impuissance. Ainsi, ni elles ni les membres de l'assistance, même lorsqu'ils sont le sujet grammatical de la phrase, n'en sont jamais l'agent : l'usage du passif, de la négation, et de suffixes pronominaux (parfois combinés) indique sans ambiguïté que l'action se fait toujours à leur détriment ${ }^{21}$. L'unique agent est cette force extérieure indéterminée à laquelle les individus ne peuvent résister - «Qu'est-ce qui agite mes mots comme un tamis », se plaint Abäğğu (6) - et qui est discrètement nommée au détour d'introductions ( «l'invité saccageur », « le terrifiant »).

Les seules actions à la portée des individus sont donc du domaine de la parole : comme le souligne le métadiscours, les chanteuses pleurent (sous-entendu, en chantant), supplient et expriment leur désarroi. Mais ces paroles elles-mêmes sont sans effet sur la fatalité qui accable cette famille : les défunts ne les entendent pas, et ne répondent pas, et jamais ne reviennent (6). Cette situation angoissante se résume en un mot, celui de « malheur » (mäkära), qui entraîne derrière lui la plainte et la résignation.

L'ensemble des stratégies rhétoriques mises en place par les chanteuses construit donc une interprétation alternative de la mort de Yəlaq que l'on peut résumer ainsi :

- Il s'agit d'une mort d'enfant, qui s'inscrit dans une série dépassant la seule personne de Yəlaq. Elle est due à une causalité naturelle, dont personne ne peut être blâmé.

- Les vivants, impuissants, ne peuvent qu'accepter cette mort et n'ont pas à exprimer de colère ni de révolte.

- Le comportement approprié est le partage de la tristesse par les partisans - parents, mais aussi amis (les seuls que Yəlaq et son frère n'aient pas nécessairement en commun).

Alors qu'elles montrent l'entrecroisement des liens personnels et rappellent aux membres de l'assemblée leurs obligations de réciprocité, les chanteuses occultent donc le geste qui a brisé ces mêmes valeurs; et tout en proclamant l'inefficacité de leurs paroles, elles entraînent donc les auditeurs dans une convergence émotionnelle où seule la tristesse a libre cours.

\section{Paroles attrapées, ou le souvenir du conflit}

51 En quoi cette interprétation alternative, construite dans l'instant des funérailles, peutelle avoir une incidence sur les événements futurs?

\section{Təzəta, se souvenir par la poésie chantée}

Comme je l'ai souligné plus haut, les distiques poétiques sont « attrapés », c'est-à-dire mémorisés, par les auditeurs; par la suite ils peuvent, dans certaines circonstances, être chantés à nouveau. Cette répétition, ou réitération, occasionne alors un phénomène de « remémoration » (təzəta $)^{22}$ : dans toute cette région, on affirme en effet que rechanter un même poème entraîne le souvenir vivace de sa performance initiale, qui surgit alors dans toutes ses composantes sonores, visuelles, et kinesthésiques - une expérience de voyage mental fortement émotionnelle (Morand, 2008). 
Ces remémorations ont lieu dans deux circonstances différentes :

- La première est celle des funérailles elles-mêmes : un distique composé pour la mort d'un individu est souvent rechanté aux funérailles d'un autre ; sa réitération, qui est précédée d'une introduction adaptée au nouveau défunt et à l'audience, contribue à l'effet de contagion des pleurs: les auditeurs, reconnaissant le poème, se souviennent de l'enterrement de leur proche et en revivent la douleur. Il est probable, par exemple, que le poème (6) d'Abäğğu provienne de l'enterrement d'une femme - peut-être Šašä, dont le nom est cité à plusieurs reprises.

- La seconde nous intéresse plus particulièrement : il s'agit de la solitude. Hommes et femmes chantent beaucoup lorsqu'ils sont seuls - les femmes chez elles lors de leurs travaux solitaires, et les hommes lorsqu'ils gardent le bétail en forêt ou en protègent, la nuit, l'enclos des voleurs potentiels. Tout distique chanté lors d'un événement social (enterrement, fête, travaux collectifs, etc.) est ainsi susceptible d'être remémoré des semaines, des mois, ou des années après. Les introductions, qui n'ont plus d'utilité pragmatique, sont alors réduites au minimum (quelques interjections).

Poème après poème et jour après jour, ces moments sont rappelés, leurs émotions ravivées, et leurs significations repensées, comme autant de fragments d'un récit de vie (Morand, 2010a).

\section{Le choix du genre et ses conséquences}

On comprend donc l'importance de ce qui est dit lors des funérailles et des choix qui y sont faits. Parmi ceux-ci, celui du genre musico-poétique est particulièrement crucial. Comme nous l'avons vu, seul l'əngurguro est chanté ce jour-ci et un autre - auquel on aurait pu s'attendre, le qärärto - est totalement absent ${ }^{23}$. Ce choix est délibéré : il a trait aux tonalités émotionnelles contrastées de ces deux genres et aux points de vue différents qu'ils offrent sur les valeurs de la parenté.

J'ai montré ailleurs (Morand, 2010b) que ces deux genres, au-delà de leurs formes poétiques et musicales, se définissaient chacun par des émotions à la fois codifiées et intensément vécues, associées à un type de rapport à autrui. Ainsi, la rhétorique mettant en scène un énonciateur impuissant et affecté est une caractéristique du genre əngurguro, tout comme l'est la mise en évidence de l'entrecroisement des liens personnels. La technique vocale pleurée, les stratégies verbales expriment ainsi les valeurs du souci mutuel et de l'interdépendance et provoquent ces effets d'amplification des pleurs et de convergence émotionnelle.

Le qärärto est bien différent: alors qu'il exalte dans sa rhétorique l'autonomie et la compétition, ce genre entraîne un sentiment dit d'« ardeur " (yägalä səmet), joyeux ou colérique selon les cas. Les chanteurs y mettent en scène les liens de parenté, mais dans l'objectif explicite de rassembler les partisans dans une action contre l'ennemi, dont la présence dans les poèmes est explicite. Le qärärto est donc également en rapport avec l'idéologie de la parenté, mais selon un point de vue sensiblement différent : ce ne sont pas les multiples liens d'entraide et d'affection qui sont affirmés, mais une fusion entre partisans en vue de l'affrontement. Selon les dires, il serait tout à fait impossible de résister à l'efficacité de ces chants: lorsqu'il est chanté dans un enterrement, par exemple, les hommes réagissent en balançant leurs fusils et en tirant en l'air. Dans le cas d'une mort naturelle, ce refus de la mort n'est guère plus qu'un hommage appuyé à la bravoure du défunt et de ses proches ; mais en cas de meurtre, il prend la forme d'un 
appel à la vengeance - une vengeance qui est parfois exécutée sur-le-champ, dans l'ébullition de la colère et de la douleur.

Qu'advient-il de ces poèmes sur le long terme et en quoi sont-ils décisifs? Deux exemples peuvent nous en donner une idée. Le premier est celui d'une femme dont deux frères étaient morts au cours d'une vendetta. Lors de mes séjours chez elle, je l'entendais - de temps à autre - chanter, pleurer et chanter des poèmes de qärärto ; à la même époque, elle faisait pression sur un jeune homme de la famille pour qu'il accomplisse la vengeance. L'adéquation entre la tonalité émotionnelle des chants, les paroles échangées avec les proches, et les décisions prises étaient tout à fait frappantes. Le second exemple est très proche du cas de Yəlaq puisqu'il s'agit d'un conflit entre frères s'étant soldé par un meurtre. La vengeance en est une solution inconcevable ; mais cela n'empêche ni la colère ni le ressentiment de se mêler à la tristesse. Dans cette histoire que j'ai évoquée ailleurs (Morand, 2010a, 2010b), un troisième frère joue un rôle important: il est celui qui a mené - mal, selon certains - le processus de réconciliation après le drame. Cet homme pleure souvent le souvenir du mort par des poèmes d'əngurguro. Mais il avait aussi chanté un qärärto lors de l'enterrement, qärärto « dangereux » qu'il s'interdisait - me disait-il - de rechanter dans la solitude. Ce n'est que le jour où la réconciliation, enlisée, lui semblait définitivement compromise que j'ai pu l'entendre, alors qu'il surveillait son bétail non loin de la maison. Pendant les longs mois où il espérait une issue au conflit, il avait maîtrisé sa colère en refusant de se souvenir du meurtre par le qärärto pour éviter d'en ressentir les effets.

\section{Yəlaq ou la tristesse en souvenir}

59 Il est donc prêté, dans cette région du Goğğam, une étonnante efficacité à la poésie chantée. Lors des funérailles de Yəlaq, le choix de ne chanter que de l'angurguro suppose, et entraîne, un certain regard sur les obligations morales des individus, ainsi que des stratégies verbales qui construisent, en l'occurrence, une version alternative de la mort et occultent le meurtre; et si on note de rares voix discordantes, comme celle de Səmačaw, celles-ci ne quittent à aucun moment le registre de l'əngurguro :

วnoraləhu bayə bet əsäraləhu bayə

Je vivrai, disait-il, je fonderai un foyer, disait-il

Yätalägmawən t'əyətun sayayə

Sans voir la balle qui avait été chargée

60 Je n'ai pas eu l'occasion de suivre les protagonistes de cette histoire, mais il est probable que la réconciliation leur est moins difficile : bien que le souvenir du meurtre de Yəlaq reste vivace, aucune autre version que celle de la tristesse et de l'acceptation n'est offerte aux remémorations musicales des individus. Après avoir écarté la colère sur un très court terme, ils pourraient bien l'apaiser sur le long terme. Dans cette histoire comme dans bien d'autres, la poésie chantée offre à un événement ses termes et sa tonalité émotionnelle, autrement dit une partie des clés pour le penser. 


\section{BIBLIOGRAPHIE}

Selon l'usage, les auteurs éthiopiens sont indexés par leur prénom, suivi du prénom de leur père.

BENDER, M. Lionel, BOWEN, J. Donald, COOPER, R. Leon, 1976, Language in Ethiopia, London, Oxford University Press.

BERHANU GEBEYEHU, 2002, Musho (dirge) as a socio-political commentary, in Baye Yiman et al. (eds), Ethiopian Studies at the End of the Second Millennium, Proceedings of the XIVth International Conference of Ethiopian Studies, Addis Abeba, Institute of Ethiopian Studies, pp. 1907-1935.

BONINI BARALDI, Filippo, 2010, L'émotion en partage. Approche anthropologique d'une musique tsigane de Roumanie, Thèse de doctorat, Université Paris Ouest Nanterre.

CoHEN, Marcel, 1924, Couplets amhariques du choa, Journal asiatique, vol. CCV, p. 1-100.

Getie Gelaye, 1998, Semantic Analysis of Amharic Kinship Terms in Gojjam: An Anthropological Approach, AFRICA Rivista trimestrale di studi e documentazione dell'Istituto italiano per l'Africa el'Oriente, vol. 53, no 1, pp. 71-92.

Getie Gelaye, 2000, Peasants and the Ethiopian State: Agricultural Producers' Cooperatives and their reflections in Amharic Oral Poetry, Hamburg, LIT.

Getie Gelaye, 2005, Engurguro, in Siegbert Uhlig (ed.), Encyclopaedia Aethiopica, vol. II, Wiesbaden, Harrassowitz Verlag, pp. 305-306.

HoBEN, Allan, 1973, Land Tenure among the Amhara of Ethiopia: The Dynamics of Cognatic Descent, Chicago, The University of Chicago Press.

HoBEN, Susan, 1976, Amhara Verbal Behavior: a commentary, Anthropological Linguistics, vol. 18, no 8 , pp. 380-386

KANE, Thomas, 1990, Amharic-English Dictionary, 2 vol., Wiesbaden, Harrassowitz.

MENGHISTU LEMMA, 1963, Yäamarəña gət'əm aynätu səratu sərəatu, Journal of Ethiopian studies, I, 2, p. 133-151.

MorAND, Katell, 2008, Chanter pour soi : La tïzïta du berger, Annales d'Éthiopie, XXIII 23, p. 45-59.

MORAND, Katell, 2010a, Fragments de vies : quand les bergers se racontent par la musique, in G. D'Agostino, M. Kilani et S. Montes (éds), Histoires de vies, témoignages, autobiographies de terrain. Formes d'énonciation et de textualisation, Munchen, Lit Verlag, p. 257-272.

MoRAND, Katell, 2010b, Vaine existence : entre deux genres, ou les effets d'un poème chanté, in C. Calame, F. Dupont, B. Lortat-Jacob et M. Manca (éds), La voix actée. Pour une nouvelle ethnopoétique, Paris, Éditions Kimè, p. 23-41.

\section{NOTES}

1. Le Goğğam, divisé en deux zones (Est et Ouest), fait partie de l'État régional Amhara. Les habitants y sont des agriculteurs-éleveurs cultivant leurs champs à l'araire; ils parlent l'amharique, une langue dite éthio-sémitique (Bender et al., 1976); et une majorité de la population se définit comme membre de l'Église Orthodoxe éthiopienne. 
2. Allan Hoben (1973) avait souligné l'existence de cette compétition dans l'ancien système de tenure.

3. Qui ne sont ni professionnels, ni rémunérés, contrairement aux usages en cours dans d'autres régions de langue amharique (Berhanu Gebeyehu, 2002).

4. En ce qui concerne la terminologie, il existe d'importantes variations régionales, et ce genre est connu ailleurs sous les noms de wäyəta, mušo, ou encore ləqso.

5. La métrique du distique (gət'əm), strictement syllabique, est bien connue (Cohen, 1924 ; Mengistu Lemma, 1963). La condensation du sens consiste, comme dans les proverbes, à dire le plus avec le moins de mots, d'où des difficultés dues à une syntaxe parfois elliptique (Hoben, 1976, 382). Enfin, Getie Gelaye (2000) et Berhanu Gebeyehu (2002) ont donné des exemples des opinions de nature politique qu'ils communiquent.

6. L'existence de ces introductions (azmač) a été jusqu'à présent complètement ignorée des recueils de poésie et des analyses littéraires.

7. Un grand merci à Beyene Abebe pour son aide dans la transcription et la traduction des poèmes présentés ici.

8. Remarque sur les noms : Yəlqal est une variante de Yəlaq, Šašätu un diminutif de Šašä.

9. Un point déjà relevé par Allan Hoben (1973, 14-17).

10. «Mon enfant » (lə̆̆g), « mes chers corps » (akalate), «ma filiation » (yäne zär), " mes frères, mes sœurs » (วhət wändəmočə), etc.

11. Par exemple, «le fils de ma mère" peut dans ce contexte désigner n'importe quelle connexion par la branche maternelle de la chanteuse, ou par la mère de Yəlaq.

12. Abäğğu (4) énumère dans son introduction un grand nombre de défunts tout en évoquant la maison de Yəlaq.

13. On peut supposer qu'en incluant dans cette figure la dizaine d'autres chanteurs, on parviendrait à une image très complète de la multiplicité des intersections entre le réseau personnel de Yəlaq et ceux des participants à ses funérailles.

14. Ce type de jeux d'énonciation semble commun dans les lamentations et chants de funérailles. Dans sa thèse de doctorat, Filippo Bonini Baraldi (2010) en dénoue et analyse ainsi un très bel exemple.

15. L'indétermination peut prendre la forme d'une troisième personne du pluriel.

16. Exemple : biyaqulbəñ, littéralement, "parce qu'ils sont morts à mes dépens », Mulu (9).

17. Exemple : «Il faut se rassembler, secouer [le souvenir] des morts », Abäğğu (1).

18. Exemple : «L'époque de Täbayən, nous la connaissions », Alämayähu (8).

19. Cette absence est elle-même étonnante, car la liturgie (fət'at) est en principe accordée à tout individu de plus de quinze ans.

20. Voir Mulu (7) qui mentionne « les gens de Monsieur Däse qui plantent des descendances ».

21. Exemples : « que je sois mangée », «parce qu'ils me sont disparus » (9).

22. Évoqué ici par Mulu (9), sous sa forme verbale: təz alläñ «je me souviens», «je me remémore ».

23. Le troisième genre, ərgədo, n'est chanté que dans les funérailles de personnes âgées ou particulièrement respectées. 


\section{RÉSUMÉS}

En décembre 2005, un petit village du Goğğam (Éthiopie) connaît un accès de tension : un conflit entre deux frères vient de se solder par un meurtre. Les funérailles s'organisent dans une atmosphère d'angoisse et d'incertitude alors que la famille se trouve prise dans la double injonction, devenue intenable, de venger la victime et de soutenir le meurtrier.

Cet article montre que les chants entonnés lors de ces funérailles, qui sont destinés à être mémorisés, réitérés et ressassés, jouent un rôle déterminant pour la suite des événements. Grâce à une analyse détaillée des choix pragmatiques et des stratégies verbales, on comprend comment les chanteurs font ici pleurer l'assistance tout en proposant une version alternative de la mort du jeune homme - une version où la violence est occultée pour ne laisser que la tristesse en souvenir.

In December 2005, a small village in Goğğam (Ethiopia) witnessed an outburst of tension: a conflict between two brothers had just lead to a murder. Funerals were held in an atmosphere of anxiety and uncertainty as the family was caught in the double injunction, untenable here, to avenge the victim and support the murderer. This article demonstrates that the songs sung at the funerals, which are intended to be memorized and repeated over and over, lay a key role in the subsequent events. Through a detailed analysis of pragmatic choices and verbal strategies, we understand how singers make the audience cry while suggesting an alternative version of the death of the young man - a version in which violence is discarded and only sadness left as a memory.

\section{INDEX}

Mots-clés : chant, funérailles, violence, remémoration, pragmatique

Keywords : Amhara, Song, Funerals, Violence, Remembering, Ethiopia, Anthropology, Pragmatic Thèmes : anthropologie (Afrique), ethnomusicologie

Index géographique : Amhara, Éthiopie 